\title{
MOTION OF SPIRALS BY CRYSTALLINE CURVATURE
}

\author{
Hitoshi Imai $^{1}$, NaOyuki Ishimura ${ }^{2}$ and TAKeo UshiJima ${ }^{3}$
}

\begin{abstract}
Modern physics theories claim that the dynamics of interfaces between the two-phase is described by the evolution equations involving the curvature and various kinematic energies. We consider the motion of spiral-shaped polygonal curves by its crystalline curvature, which deserves a mathematical model of real crystals. Exploiting the comparison principle, we show the local existence and uniqueness of the solution.
\end{abstract}

AMS Subject Classification. 80A22, 34A12, 52C99.

Received: March 2, 1998. Revised: October 2, 1998.

\section{INTRODUCTION}

The analysis on the growth and the motion of interfaces between the two-phase is an important problem in material sciences.

Modern physics theories developed in [11] state that the dynamics of the interface is described by the equation

$$
b(\nu) V=\left(f(\nu)+f^{\prime \prime}(\nu)\right) K-F .
$$

Here $V$ and $K$ denote the normal velocity and the curvature of the interface, respectively. $\nu$ is the angle to the interface normal, $F$ is the difference in bulk energy between two phases, $b(\nu)>0$ is the kinetic coefficient, and $f(\nu)$ is the interfacial energy. If the energy $f(\nu)$ is merely piecewise smooth and has the convexified Frank diagrams that are polygonal, which is referred to as the crystalline energy, then (1) reduces to the evolution of polygonal interfaces. Taylor [24] and independently Angenent and Gurtin [2] introduced such an evolution law, which is now called a crystalline motion or an evolution by crystalline curvature. This law is defined for a special class of piecewise linear closed curves, called admissible, and for this class, the motion is governed by a system of ordinary differential equations (ODEs). We refer to a fundamental monograph by Gurtin [11]. However, roughly speaking, most researches have been made from the following two major viewpoints. One is to study the evolution of closed curves, partly because of the connection with the so-called level set methods in mind. See $[9,13,28]$. The other is to deal with the motion of a graph. See $[3,4,6,10]$ and the references therein in this respect. Utilizing the general theory of abstract analysis or the property of variational structure, the authors of the latter articles obtain fairly satisfactorily results.

\footnotetext{
Keywords and phrases. Crystalline motion, spiral-shaped polygonal curves, material sciences.

1 Department of Applied Physics and Mathematics, Faculty of Engineering, University of Tokushima, Tokushima 770-8506, Japan. e-mail: imai@pm.tokushima-v.ac.jp

2 Department of Mathematics, Hitotsubashi University, Kunitachi, Tokyo 186-8601, Japan.

3 Graduate School of Mathematical Sciences, University of Tokyo, Komaba, Tokyo 153-8914, Japan.
} 
In this paper, on the other hand, we investigate the motion of spiral-shaped curves by their crystalline curvature. The curves we consider spiral out to infinity, with one end being fixed at the origin; they are not represented by the level set of a scalar function nor a graph. For these curves, however, the governing ODE system becomes infinite order and the comparison principle established in [7] does not seem to hold. We circumvent these points by an approximation argument and applying the comparison principle to these approximated equations. Our aim is then to prove the short-time existence of solutions to the original infinite system of ODEs. We also show the uniqueness of the solution for this system.

The spiral-shaped crystals are known to exist in reality. For example, we recall mono-molecular spirals observed on the (0001) face of $\mathrm{SiC}[16,18,22]$. These spirals are reported to obey the normal velocity equation (1) (see $[16,18]$ ). One of our motivations of research is to model these fascinating patterns mathematically rigorously. As a first attempt, we assume that the underlying Wullf diagram of our spirals is a regular polygon and consider the case $b=1, F=0$ and $\mathrm{f}$ being piecewise unity, for simplicity. For details, see the assumptions (A1), (A2) and (A3) in Section 2. Furthermore, we focus our attention on the existence of solutions up to the first singularity. Even under these settings, the problem is technically complicated and our results seem to be new. Numerical investigations are made on the spirals with different conditions, which shows various aspects of our problem.

We end this introduction with recalling briefly the spirals appeared in other areas, for completeness of our exposition. It is well known that spiral patterns are commonly observed in a variety of biological and chemical systems; perhaps the most famous one is presented in the Belousov-Zhabotinskii reagent. Much attention has been paid to understand these phenomena both numerically and analytically, based on various mathematical models. See for instance $[12,15,17,27]$ and the references therein. Compared to these, the mathematical studies on spirals in crystals seem to be rarely attempted [26]. We remark that the simplest curvature evolution, which is known as the curve shortening equation, also produces spiral-shaped solutions [14].

With regard to an application of the method of crystalline algorithm to other problems, we refer for instance to a recent work by Almgran [1]. For higher dimensional case, see [8].

The organization of this paper is as follows. In Section 2 we formulate the problem and state our main results. In Section 3 we prove Theorem 1. Some numerical examples are exhibited in Section 4 . We discuss further problems in Section 5.

\section{Formulation And RESUlts}

We begin with basic formulations on the motion of polygonal curves by crystalline curvature. We mainly follow the expositions in $[20,21,25]$, to which we refer for more detail.

Suppose the spiral-shaped polygonal curve $S(t)$ is given for each $t$ in some interval. Without loss of generality, we assume that $S(t)$ spirals out counterclockwise. Let $L_{0}(t), L_{1}(t), L_{2}(t), \cdots, L_{i}(t), \cdots$ be the line segments of $S(t)$, numbering from the origin and let $l_{i}(t)$ denote the length of $L_{i}(t)$. The origin is kept fixed during the evolution. The unit normal vector $\nu_{i}$ to $L_{i}(t)$ will be oriented outwardly and independent of $t$. The set $\mathcal{N}$ of possible orientation $\nu_{i}$ is finite, which appears in the so-called Wulff crystal [23]; that is, the unique crystal which minimizes the surface energy at the fixed enclosed area. We remark that $L_{i}(t)$ is contained in $\left\{x \in \mathbb{R}^{2} \mid x \cdot \nu_{i}=d_{i}(t)\right\}$, where $d_{i}(t)$ represents the distance from the origin to the line containing $L_{i}(t)$. Moreover, we restrict our attention to the case that $\nu_{i+1}$ is attained by turning $\nu_{i}$ left for every $i$. (See Fig. 1.)

The crystalline curvature $K_{i}(t)$ on each $L_{i}(t)$ is now formulated as

$$
K_{i}(t)=\frac{1}{l_{i}(t)}\left(\left(\cot \theta_{i}+\cot \theta_{i+1}\right)-\frac{1}{\sin \theta_{i}}-\frac{1}{\sin \theta_{i+1}}\right)
$$

where the angle $\theta_{i}$ is defined by the one between $\nu_{i-1}$ and $\nu_{i} ;$ i.e., $\cos \theta_{i}=\nu_{i-1} \cdot \nu_{i}$. For the derivation, we refer to the equation (12) of [11]. It is well-known that $K_{i}(t)$ is the derivative of the length with respect to the area under the normal motion of entire edges [20,24], and hence naturally extends the notion of the "smooth" 


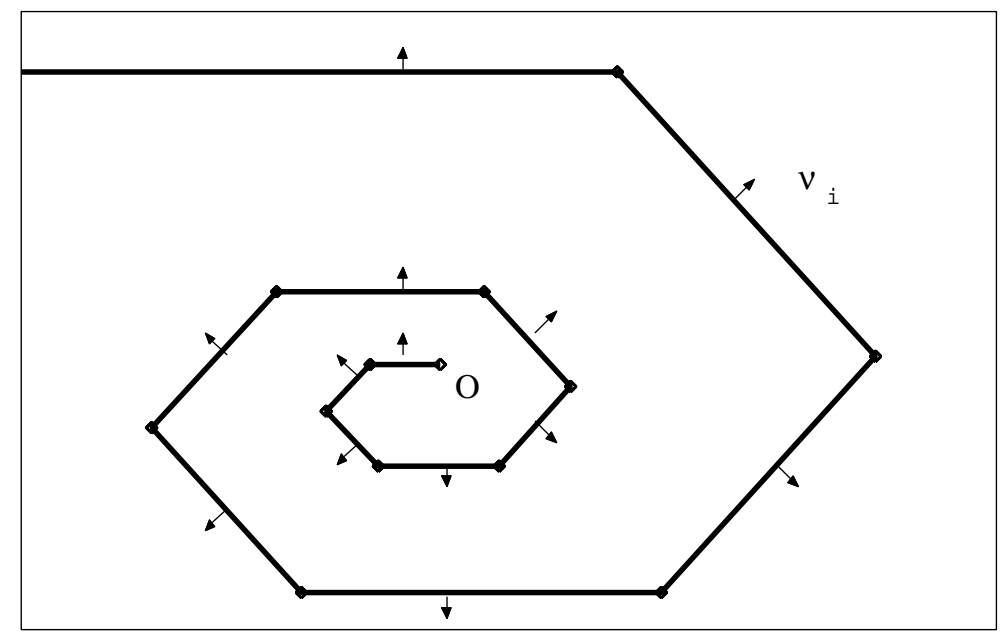

Figure 1. Example of spiral shaped polygonal curve.

curvature. Since the geometry yields

$$
l_{i}(t)=-d_{i}(t)\left(\cot \theta_{i}+\cot \theta_{i+1}\right)+\frac{d_{i-1}(t)}{\sin \theta_{i}}+\frac{d_{i+1}(t)}{\sin \theta_{i+1}},
$$

the basic equation of the crystalline motion is then reduced to the ODE system for $d_{i}(t)$. Namely, the equation (1) with $b=1, F=0$ and $f$ being piecewise unity is reduced to

$$
\begin{aligned}
\frac{\mathrm{d}}{\mathrm{dt}} d_{i}(t) & =K_{i}(t), \\
& =-\frac{\left(\cot \theta_{i}+\cot \theta_{i+1}\right)-1 / \sin \theta_{i}-1 / \sin \theta_{i+1}}{-d_{i}(t)\left(\cot \theta_{i}+\cot \theta_{i+1}\right)+d_{i-1}(t) / \sin \theta_{i}+d_{i+1}(t) / \sin \theta_{i+1}} .
\end{aligned}
$$

We remark that $d_{0}(t) \equiv 0$ in our setting. If we differentiate (4) once, we obtain the equivalent system for the unknowns $K_{i}(t)$.

$$
\begin{aligned}
\frac{\mathrm{d}}{\mathrm{dt}} K_{i}(t)=K_{i}(t)^{2}\left(\frac{1}{\sin \theta_{i}}\right. & \left.+\frac{1}{\sin \theta_{i+1}}-\cot \theta_{i}-\cot \theta_{i+1}\right)^{-1} \\
\times & \left(\frac{K_{i-1}(t)}{\sin \theta_{i}}+\frac{K_{i+1}(t)}{\sin \theta_{i+1}}-K_{i}(t)\left(\cot \theta_{i}+\cot \theta_{i+1}\right)\right) .
\end{aligned}
$$

We will rather discuss this system in course of proof. See (8) below.

To proceed further, we impose the following assumptions (A1, A2, A3) which our spirals should satisfy.

(A1) The angle $\theta_{i}$ between $\nu_{i}$ and $\nu_{i-1}$ identically equals $2 \pi / n$ for all $i$, where $n$ is a positive integer.

(A2) $d_{i}(0) \leq d_{i+1}(0)$ for all $i$ and the equality does not hold for every consecutive $n$; i.e., we have $d_{i}(0)<d_{i+n}(0)$. Further, $\lim _{i \rightarrow \infty} d_{i}(0)=\infty$.

(A3) $\liminf _{i \rightarrow \infty} l_{i}(0)=\infty$. The assumption (A1) means that the set $\mathcal{N}$ of the normals is essentially the same one as regular $n$-polygons. (A1) should be fulfilled under the evolution, while (A2) and (A3) are the initial conditions. We remark that (A1) and (A2) make the ODE system (3) infinite order. In view of $K_{i}(t) \leq 0$ 
for all $i$ [see (2)], we easily see that (A3) is equivalent to

(A3)' $\liminf _{i \rightarrow \infty} K_{i}(0)=0$.

Now we state our main result.

Theorem 1. Assume (A1, A2, A3). Then the initial value problem for the ODE system (5) is uniquely solvable. This solution develops a singularity in finite time.

We conclude this section with remarks concerning the assumptions. (A2) and (A3) do not necessarily imply the monotonicity of $l_{i}(0)$. It may happen that $l_{i}(0)>l_{i+n}(0)$. Moreover, we can devise a spiral satisfying $(\mathrm{A} 1)$ through (A3) and

$$
\inf _{i}\left(d_{i+n}(0)-d_{i}(0)\right)=\lim _{i \rightarrow \infty}\left(d_{i+n}(0)-d_{i}(0)\right)=0
$$

Nevertheless, (A2) and (A3) suffice to show the solvability of the initial value problem. Next a loop is able to be formed without the strict inequality about $d_{i}(0)$ for the consecutive $n$; a loop would require $d_{i}(0)>d_{i+n}(0)$ (see Fig. 3). We investigate this situation further in numerical examples.

\section{Proof of theorem}

To prove our results we employ an approximation argument. Fix some integer $N$ and we put $l_{N}^{N}(t)=\infty$, where the superscript indicates the finite truncation. We consider

$$
\left\{\begin{array}{l}
\frac{\mathrm{d}}{\mathrm{dt}} d_{i}^{N}(t)=-\frac{-\left(\cot \theta_{i}+\cot \theta_{i+1}\right)+1 / \sin \theta_{i}+1 / \sin \theta_{i+1}}{-d_{i}^{N}(t)\left(\cot \theta_{i}+\cot \theta_{i+1}\right)+d_{i-1}^{N}(t) / \sin \theta_{i}+d_{i+1}^{N}(t) / \sin \theta_{i+1}}, \quad(i=1, \cdots, N-1), \\
d_{N}^{N}(t)=d_{N}(0), \\
d_{0}^{N}(t)=0 \\
d_{i}^{N}(0)=d_{i}(0), \quad(i=1, \cdots, N-1) .
\end{array}\right.
$$

From assumption (A1), the first equation of (7) becomes

$$
\frac{\mathrm{d}}{\mathrm{d} t} d_{i}^{N}(t)=-\frac{1}{\left(\Delta_{\theta} d\right)_{i}^{N}(t)+d_{i}^{N}(t)}, \quad i=1, \cdots, N-1,
$$

where $\theta=2 \pi / n$ and

$$
\left(\Delta_{\theta} d\right)_{i}:=\frac{d_{i+1}-2 d_{i}+d_{i-1}}{2(1-\cos \theta)} .
$$

Describing the problem in terms of the crystalline curvature $K_{i}^{N}$, we have the approximated problem for $(6)$.

$$
\left\{\begin{array}{l}
\frac{\mathrm{d}}{\mathrm{d} t} K_{i}^{N}(t)=\left(K_{i}^{N}(t)\right)^{2}\left(\left(\Delta_{\theta} K\right)_{i}^{N}(t)+K_{i}^{N}(t)\right), \quad(i=1, \cdots, N-1) \\
K_{0}^{N}(t)=0 \\
K_{N}^{N}(t)=0 \\
K_{i}^{N}(0)=K_{i}(0), \quad(i=1, \cdots, N-1) .
\end{array}\right.
$$

Note that we have imposed $d_{-1}^{N}=-\infty$ in order to be consistent with the equation (4).

Throughout this section, we use the next elementary comparison lemma. Although, to our knowledge, there seems to be no explicit presentation in the literature, we believe that the property itself is known to specialists. 
Lemma 1. Suppose there exists $t_{1}>0$ such that $a_{j}(t)$ and $b_{j}(t)$ are continuous functions for $0<i<N$, $0 \leq t \leq t_{1}$, and $a_{i}(t)>0$ for $0<i<N, 0 \leq t \leq t_{1}$. If there holds for $u_{i}(t) \in C^{1}$

$$
\begin{cases}\frac{\mathrm{d}}{\mathrm{d} t} u_{i}(t) \geq a_{i}(t)\left(\Delta_{\theta} u\right)_{i}(t)+b_{i}(t) u_{i}(t), & 0<i<N, \quad 0 \leq t \leq t_{1}, \\ u_{0}(t) \geq 0, & 0 \leq t \leq t_{1} \\ u_{N}(t) \geq 0, & 0 \leq t \leq t_{1} \\ u_{i}(0) \geq 0, & 0<i<N\end{cases}
$$

then we have

$$
u_{i}(t) \geq 0, \quad 0 \leq i \leq N, \quad 0 \leq t \leq t_{1} .
$$

We give a sketch of proof for completeness.

Proof of Lemma 1. Assume that $u_{i}(t)$ takes negative value. We set $w_{i}(t):=e^{C t} u_{i}(t)$. Here, $C$ is a constant which satisfies $C+b_{i}(t)<0$ for all $i$ and $0 \leq t \leq t_{1}$. From the assumption $w_{i}(t)$ attains negative minimum at some point $\left(i_{0}, t_{0}\right) ; 0<i_{0}<N$ and $0<t \leq t_{1}$. At this point, we have

$$
\frac{\mathrm{d} w_{i_{0}}}{\mathrm{~d} t}\left(t_{0}\right) \geq a_{i_{0}}\left(t_{0}\right)\left(\Delta_{\theta} w\right)_{i_{0}}\left(t_{0}\right)+\left(C+b_{i_{0}}\left(t_{0}\right)\right) w_{i_{0}}\left(t_{0}\right)
$$

We also have

$$
\frac{\mathrm{d} w_{i_{0}}}{\mathrm{~d} t}\left(t_{0}\right) \leq 0, \quad a_{i_{0}}\left(t_{0}\right)\left(\Delta_{\theta} w\right)_{i_{0}}\left(t_{0}\right) \geq 0
$$

and

$$
\left(C+b_{i_{0}}\left(t_{0}\right)\right) w_{i_{0}}\left(t_{0}\right)>0 .
$$

These inequalities lead a contradiction. Hence $u_{i}(t) \geq 0$ for $0 \leq i \leq N$ and $0 \leq t \leq t_{1}$.

Using above comparison lemma and arguing just like as in [5], we can easily show that the solution for (8) blows-up in finite time.

Lemma 2. There exists $T<\infty$ independent of $N$ such that $\lim _{t \rightarrow T} \max _{i}\left|K_{i}^{N}(t)\right|=\infty$.

Proof of Lemma 2. For sufficiently small positive $c$, we define

$$
\varphi_{i}:=\left\{\begin{array}{lr}
-c \cos (i \theta / 2), & 0 \leq i \leq n \\
0, & i>n .
\end{array}\right.
$$

From the comparison lemma, we have

$$
K_{i}^{N}(t)<\varphi_{i}, \quad i \geq 0, \quad t>0 .
$$

We define $J^{N}(t)$ as follows,

$$
J^{N}(t):=\sum_{i=1}^{n-1} \frac{\varphi_{i}}{K_{i}^{N}(t)}
$$


Differentiating $J^{N}(t)$, we obtain the following estimate,

$$
\begin{aligned}
\frac{\mathrm{d}}{\mathrm{d} t} J^{N}(t) & =-\sum_{i=1}^{n-1}\left(\Delta_{\theta} K^{N}+K^{N}\right)_{i} \varphi_{i} \\
& =-\sum_{i=1}^{n-1}\left(\Delta_{\theta} \varphi+\varphi\right)_{i} K_{i}^{N}-\frac{K_{n}^{N} \varphi_{n-1}}{2(1-\cos \theta)} \\
& \leq-\lambda \sum_{i=1}^{n-1} \varphi_{i}^{2}
\end{aligned}
$$

where $\lambda:=\frac{\cos (\theta / 2)-\cos \theta}{1-\cos \theta}>0$. From this inequality we can easily obtain the upper bound for the blow-up time $T$,

$$
T \leq \frac{J^{N}(0)}{\lambda \sum_{i=1}^{n-1} \varphi_{i}^{2}}
$$

Since $J^{N}(0)$ does not depend on $N$, we have proved this lemma.

We can estimate the duration of evolving spiral $S(t)$ from below. From assumption (A3)' this lower bound is seen to be independent of $N$.

Lemma 3. There exists $T_{0}$ independent of $N$ such that spiral $S(t)$ does not collapse for $t \in\left[0, T_{0}\right)$. More precisely, $l_{i}^{N}(t)$ and $d_{i+n}^{N}(t)-d_{i}^{N}(t)$ are positive in this time interval. Moreover, $T_{0}$ does not depend on $N$.

Proof of Lemma 3. We need to classify possible singularities. There can be three types of singularities:

1. Blow-up of the crystalline curvature; i.e. $\max _{i}\left|K_{i}^{N}(t)\right| \rightarrow \infty$.

2. Extinction of the 0 -th segment; i.e. $l_{0}^{N}(t) \stackrel{i}{\rightarrow} 0$.

3. A segment touches to another segment which is not adjacent; i.e. $\min _{i}\left(d_{i+n}^{N}(t)-d_{i}^{N}(t)\right) \rightarrow 0$.

We note that the type 3 singularity should be meant solely for the truncated system. It is not the property at infinity. Observe the remark after Theorem 1. By the comparison lemma, we can estimate the blow-up time of $K_{i}^{N}(t)$ from below. Taking account of assumption (A3)', we deduce this lower bound is independent of $N$. In fact, comparing $K_{i}^{N}(t)$ with the constant solution $\bar{K}_{i}(t)$ starting from $\bar{K}_{i}(0):=\inf _{i} K_{i}(0)$, we can show that there exists a small period $\left[0, T_{1}\right)$ such that $K_{i}^{N}(t)$ does not blow up in this interval.

The length of 0 -th segment $l_{0}^{N}(t)$ should satisfy

$$
\frac{\mathrm{d}}{\mathrm{d} t} l_{0}^{N}(t)=K_{1}^{N}(t) \sin \theta
$$

Because $K_{1}^{N}(t)$ is bounded for $t \in\left[0, T_{1} / 2\right]$, we can estimate the extinction time of $l_{0}^{N}(t)$ from below and this lower bound does not depend on $N$. We denote this lower bound by $T_{2}$.

It is obvious that $d_{1}^{N}(t)>0$ for $t \in\left[0, T_{2} / 2\right]$ and $d_{N}^{N}(t)$ is positive for all $t>0$. Thanks to the semidiscretized version of the maximum principle, we obtain $d_{i}^{N}(t)>0(i=2, \cdots, N-1)$ on $\left[0, T_{2} / 2\right]$. In fact, we set $h_{i}(t)=c e^{-k t}$, for $0 \leq i \leq N$, where $c$ is a positive constant with $c<\min _{0 \leq t \leq T_{2} / 2} d_{1}^{N}(t)$ and $k$ is a positive 
constant. Then we have

$$
\left\{\begin{array}{lll}
d_{i}^{N}(0)>h_{i}(0), & 1 \leq i \leq N, \\
d_{0}^{N}(t)>h_{0}(t), & 0 \leq t \leq T_{2}, & \\
d_{N}^{N}(t)>h_{N}(t), & 0 \leq t \leq T_{2}, & \\
\frac{\mathrm{d}}{\mathrm{d} t} d_{i}^{N}(t)=-\frac{1}{\left(\Delta_{\theta} d\right)_{i}^{N}(t)+d_{i}^{N}(t)}, & 0 \leq t \leq T_{2} / 2, \quad 1<i<N,
\end{array}\right.
$$

from which we find there exists $T(k)>0$ such that

$$
\frac{\mathrm{d}}{\mathrm{d} t} h_{i}(t)+\frac{1}{\left(\Delta_{\theta} h\right)_{i}(t)+h_{i}(t)}<0, \quad 0 \leq t \leq T(k) .
$$

We can choose $k$ so that $T(k)>T_{2} / 2$. If there is a point $\left(i_{0}, t_{0}\right)$ such that $0=\min _{i}\left(d_{i}^{N}\left(t_{0}\right)-h_{i}\left(t_{0}\right)\right)=$ $d_{i_{0}}^{N}\left(t_{0}\right)-h_{i_{0}}\left(t_{0}\right)$ and $d_{i}^{N}(t)>h_{i}(t)$ for $t<t_{0}$. Then $v_{i}(t):=d_{i}^{N}(t)-h_{i}(t)$ satisfy $v_{i_{0}}\left(t_{0}\right)=0, \frac{d}{d t} v_{i_{0}}\left(t_{0}\right) \leq 0$. Since we may assume $\left(\Delta_{\theta} v\right)_{i_{0}}\left(t_{0}\right)+v_{i_{0}}\left(t_{0}\right)>0$, there holds

$$
0 \geq \frac{\mathrm{d}}{\mathrm{d} t} v_{i_{0}}\left(t_{0}\right)=\frac{1}{l_{i_{0}}^{N}\left(t_{0}\right)\left(\left(\Delta_{\theta} h\right)_{i_{0}}\left(t_{0}\right)+h_{i_{0}}\left(t_{0}\right)\right)}\left(\left(\Delta_{\theta} v\right)_{i_{0}}\left(t_{0}\right)+v_{i_{0}}\left(t_{0}\right)\right)>0
$$

which is a contradiction. We conclude that $d_{i}^{N}(t)>h_{i}(t)$ for $0 \leq i \leq N, 0 \leq t \leq T_{2} / 2$.

Let $\delta_{i}(t):=d_{i+n}^{N}(t)-d_{i}^{N}(t)$, then

$$
\begin{cases}\frac{\mathrm{d}}{\mathrm{d} t} \delta_{i}(t)=\frac{1}{K_{i}^{N} K_{i+n}^{N}}\left(\left(\Delta_{\theta} \delta\right)_{i}(t)+\delta_{i}(t)\right), & i=1, \cdots, N-n-1 \\ \delta_{0}(t)>0 & \\ \delta_{N-n}(t)>0, & i=1, \cdots, N-n-1 \\ \delta_{i}(0)>0, & \end{cases}
$$

From the comparison lemma, we infer $\delta_{i}(t)>0$ on $\left[0, T_{2} / 2\right]$. Hence we can exclude the third possibility. By setting $T_{3}:=\min \left\{T_{1} / 2, T_{2} / 2\right\}$ we have proved the local existence of the solution. Finally, we extend this time interval to the maximal one $\left[0, T_{0}\right)$.

\section{Proof of Theorem 1.}

1. Existence. We extend $K_{i}^{N}(t)$ for $i>N$ by 0 ; that is, we define $K_{i}^{N}(t)=0$ for $i>N$. Let $k_{i}(t):=$ $K_{i}^{M}(t)-K_{i}^{N}(t)(M>N)$, then $k_{j}(t)$ satisfy

$$
\frac{\mathrm{d}}{\mathrm{d} t} k_{i}(t)=\left(K_{i}^{M}(t)\right)^{2}\left(\Delta_{\theta} k\right)_{i}(t)+\left\{\left(K_{i}^{M}(t)+K_{i}^{N}(t)\right)\left(\left(\Delta_{\theta} K\right)_{i}^{N}(t)+K_{i}^{N}(t)\right)+\left(K_{i}^{M}(t)\right)^{2}\right\} k_{i}(t) .
$$

From Gronwall's inequality we obtain for $t \in\left[0, T_{0} / 2\right]$

$$
\|k(t)\| \leq C\|k(0)\|
$$

where we denote $\left\{k_{i}(t)\right\}$ by $k(t)$ and $\|k\|:=\sup _{0 \leq i \leq M}\left|k_{i}\right| . T_{0}$ is the same as in Lemma 3 . We note that a constant $C$ depends only on the bounds of $K_{i}^{N}(t)$ and $K_{i}^{M}(t)$. However, these bounds do not depend on $N$ and $M$ in view of Lemma 3, which implies that $C$ is independent of $N$ and $M$. By way of approximation and the assumption (A3), we have

$$
\lim _{N, M \rightarrow \infty}\|k(0)\|=0
$$



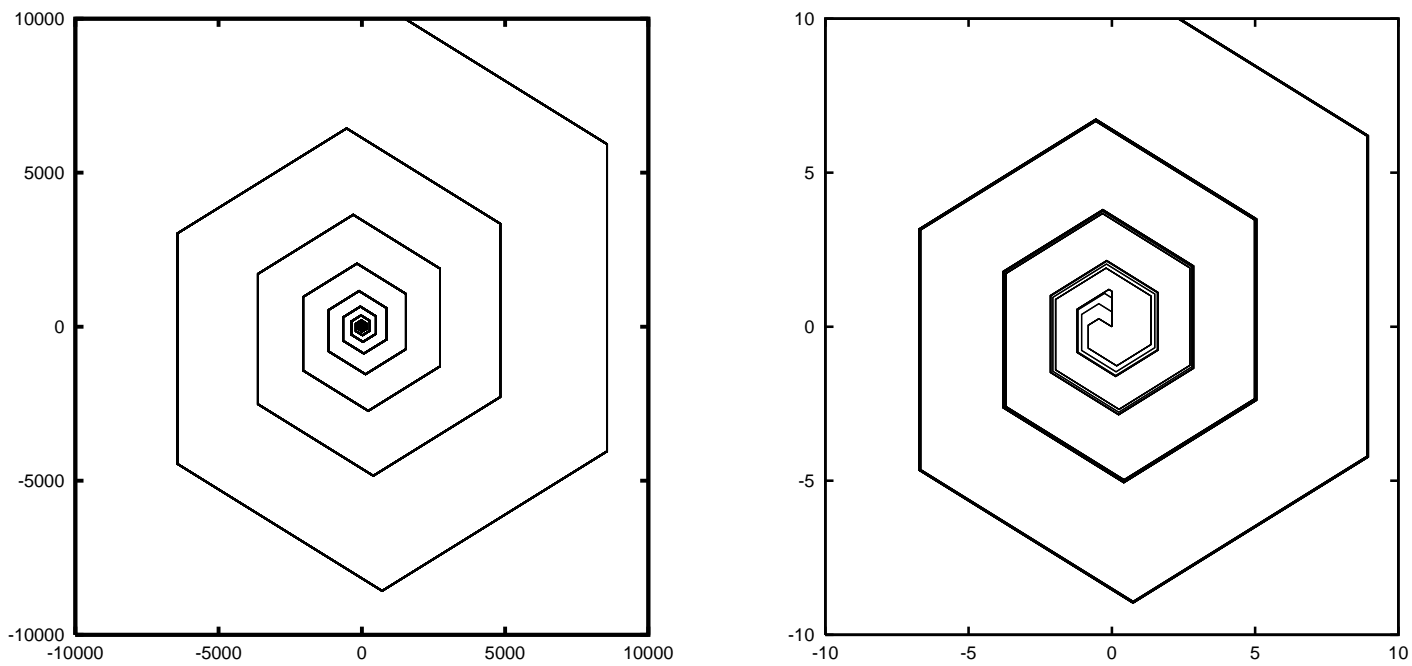

FIGURE 2. Evolution of spiral-shaped polygonal curve by crystalline curvature.

Tending $N$ and $M$ to infinity in inequality (9), we see that $\left\{K_{i}^{N}(t)\right\}$ is a Cauchy sequence. By using the same argument for $\mathrm{d} k_{i} / \mathrm{d} t$, we can show that there exist limit functions $K_{i}^{\infty}(t)$ such that

$$
\lim _{N \rightarrow \infty} K_{i}^{N}=K_{i}^{\infty} \quad \text { in } C^{1}\left(\left[0, T_{0} / 2\right]\right)
$$

Therefore, $\left\{K_{i}^{\infty}(t)\right\}$ satisfies (6) for $t \in\left[0, T_{0} / 2\right]$. Extending to the maximal time interval, we have proved the existence part of Theorem 1 .

2. Uniqueness. Suppose there exist two solutions $K_{i}(t)$ and $K_{i}^{\prime}(t)$ for $(6)$. For fixed $N$ and $T>0$ we may assume that for some $0<i_{0} \leq N, \max _{0<i<N, 0 \leq t \leq T}\left(K_{i}(t)-K_{i}^{\prime}(t)\right)=\left(K_{i_{0}}\left(t_{0}\right)-K_{i_{0}}^{\prime}\left(t_{0}\right)\right)>0$. From the semidiscretized version of maximum principle, $i_{0}=N$. Since $N$ is arbitrary, we conclude that $K_{i}(t)=K_{i}^{\prime}(t)$ for all $i$ from the boundary condition at $i=\infty$.

\section{Numerical EXAMPLES}

In this section we show several numerical examples. For this numerical simulation we employ Euler discretization of equation (8). As to the detailed numerical analysis under other settings, we refer to [19].

The first example deals with the situation described in Theorem 1. From Figure 2, we can see that the facets, which are far from the origin, almost never move. The 0th segment is seen to shrink first. In this case, we can continue our process numerically after the singularity by renumbering $i+1$ to $i$.

In the second example, the initial spirals does not satisfy the assumption (A2) and has a loop. The existence of a loop seems unrealistic and it may give just a mathematical toy model, which shows the justification of (A2) in a sense. In this situation, anyway, singularity develops by extinction of this loop, provided the initial loop is small enough. See Figure 3.

In the third example, we drop the assumption (A1); the angles $\theta_{i}$ do not necessarily coincide with the single value. This means that the corresponding crystals exhibit anisotropy, whose mathematical analysis seems to become much complicated. See Figure 4. 

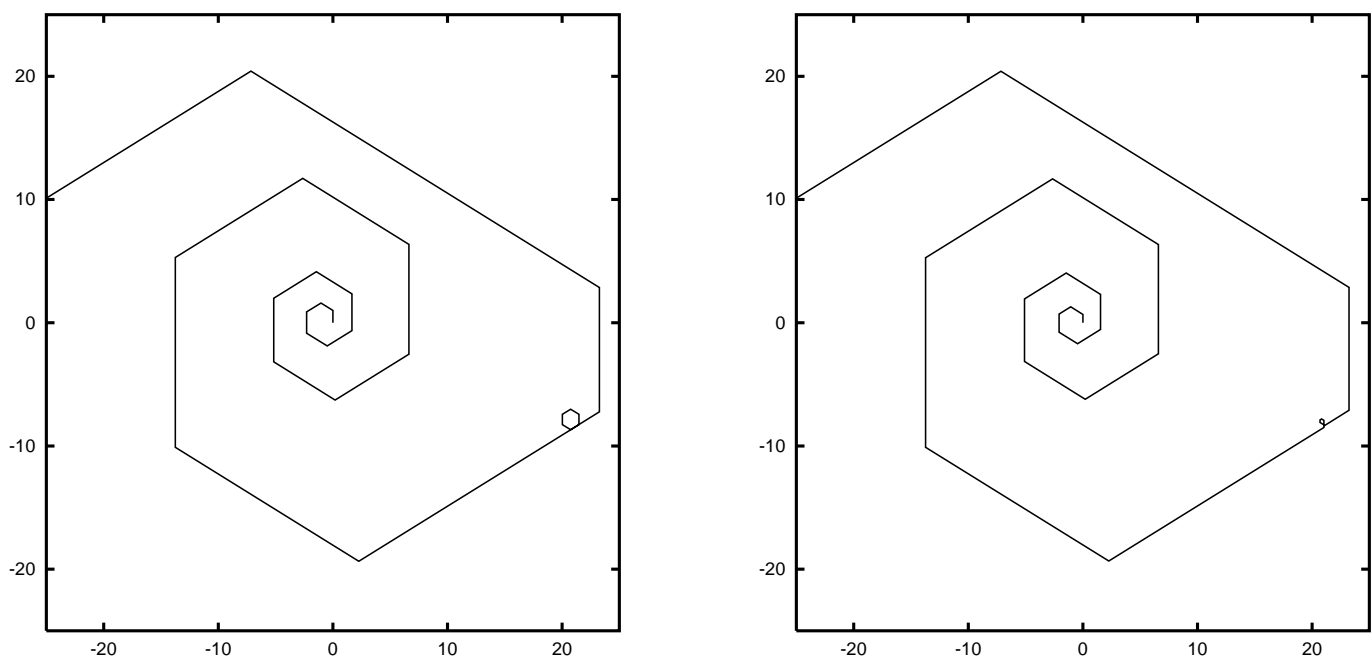

FiguRE 3. Initial spiral has a small loop.
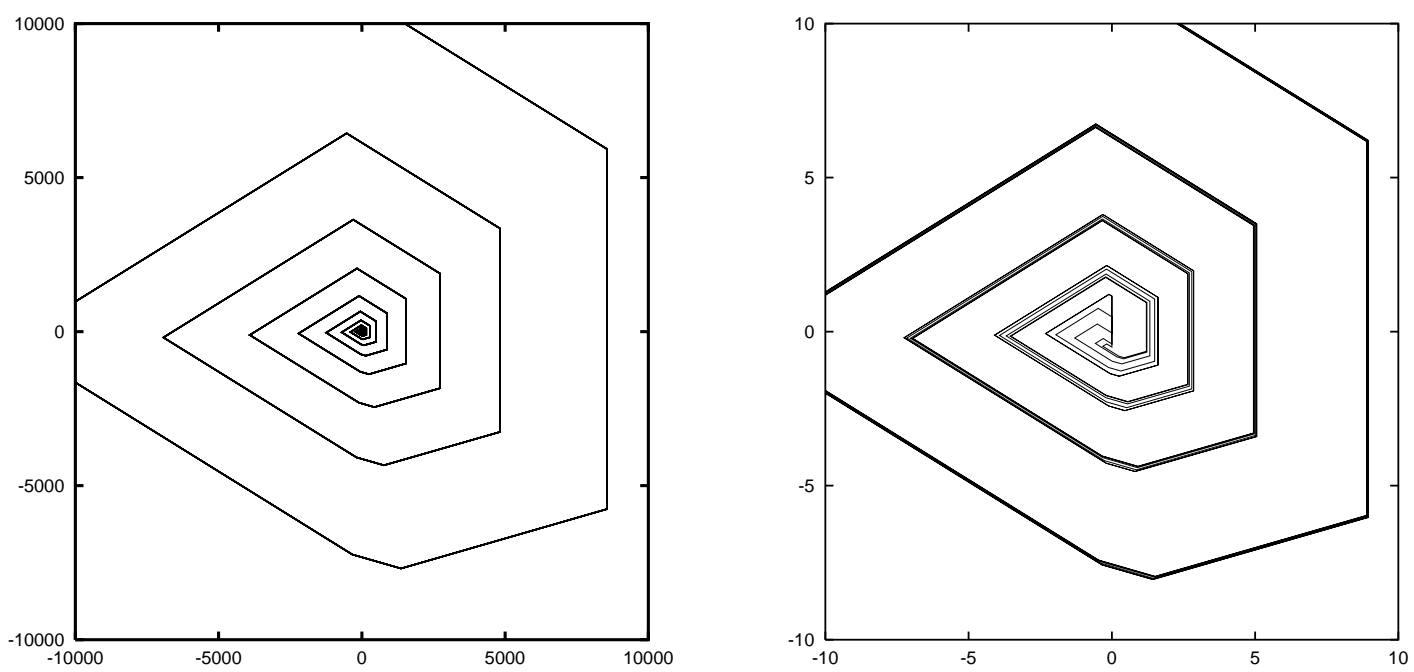

FIGURE 4. Wulff diagram is not a regular polygon.

\section{Discussion}

We have investigated the evolution of spiral-shaped polygonal curves by its crystalline curvature in its simplest case. The short term existence of solutions to the infinite order ordinary differential equations is shown via the approximation and the comparison principle to the truncated system. The uniqueness of these solutions is also proved. The case that the Wulff diagram is a regular polygon is treated [observe the assumption (A1)]; our methods seem to need modifications in order to apply it directly to other cases. We remark that the articles which treat the motion of a graph as well as reference [7] do not impose such restriction concerning the Wulff diagram. Unfortunately, these results seem to require generalizations in order to be directly applicable to our problem. Moreover, in real experiments, a container would be present and hence the infiniteness of our ODE system seems unrealistic. In this regard, our finite approximation schemes may be interpreted as a natural model, since the part of curves far from the origin would have little effect on the whole dynamics. 
We do not analyze the formation of spiral patterns; in our setting, spiral shaped polygonal curves are given from the beginning. It should be our further topics for investigation to understand the mechanics of the growth of crystals via the crystalline curvature algorithm. See also $[16,18]$.

The third author (TKU) would like to express his gratitude to Professor Hiroshi Matano for guidance and encouragement. We are grateful to Dr. MiHo Giga for her interest in this research. Thanks are also due to the referees for helpful comments and suggestions. This work is partially supported by Grants-in-Aid for Scientific Research (No. 09304023, 09354001, 09740139, 09440080), Japan Ministry of Education, Science, Sports and Culture.

\section{REFERENCES}

[1] R. Almgren, Crystalline Saffman-Taylor fingers. SIAM J. Appl. Math. 55 (1995) 1511-1535.

[2] S. Angenent and M.E. Gurtin, Multiphase thermodynamics with interfacial structure 2. Evolution of an isothermal interface. Arch. Rational Mech. Anal. 108 (1989) 323-391.

[3] C.M. Elliott, A.R. Gardiner and R. Schätzle, Crystalline curvature flow of a graph in a variational setting. Adv. Math. Sci. Appl. 8 (1998) 425-460.

[4] T. Fukui and Y. Giga, Motion of a graph by nonsmooth weighted curvature, in World Congress of Nonlinear Analysis '92, Vol. I, V. Lakshmikantham Ed., Walter de Gruyter, Berlin (1996) 47-56.

[5] M.E. Gage, On the size of the blow-up set for a quasilinear parabolic equation. Contemp. Math. 127 (1992) 41-58.

[6] M.-H. Giga and Y. Giga, Evolving graphs by singular weighted curvature. Arch. Rational Mech. Anal. 141 (1998) $117-198$.

[7] Y. Giga and M.E. Gurtin, A comparison theorem for crystalline evolution in the plane. Quart. Appl. Math. 54 (1996) $727-737$.

[8] Y. Giga, M.E. Gurtin and J. Matias, On the dynamics of crystalline motion. Japan J. Indust. Appl. Math. 15 (1998) 7-50.

[9] P.M. Girão, Convergence of a crystalline algorithm for the motion of a simple closed convex curve by weighted curvature. SIAM J. Numer. Anal. 32 (1995) 886-899.

[10] P.M. Girão and R.V. Kohn, Convergence of a crystalline algorithm for the heat equation in one dimension and for the motion of a graph by weighted curvature. Numer. Math. 67 (1994) 41-70.

[11] M.E. Gurtin, Thermomechanics of evolving phase boundaries in the plane. Oxford, Clarendon Press (1993).

[12] R. Ikota, N. Ishimura and T. Yamaguchi, On the structure of steady solutions for the kinematic model of spiral waves in excitable media. Japan J. Indust. Appl. Math. 15 (1998) 317-330.

[13] K. Ishii and H.M. Soner, Regularity and convergence of crystalline motion. Preprint (1996).

[14] N. Ishimura, Shape of spirals. Tôhoku Math. J. 50 (1998) 197-202.

[15] W. Jahnke and A.T. Winfree, A survey of spiral-wave behaviors in the oregonator model. Internat. J. Bifur. Chaos 1 (1991) $445-466$.

[16] T. Kuroda, Kessyou ha ikiteiru (Crystal is alive). Science Sya, Tokyo (1984).

[17] A.S. Mikhailov, V.A. Davydov and A.S. Zykov, Complex dynamics of spiral waves and motion of curves. Physica D 70 (1994) $1-39$.

[18] A. Ookawa, Kessyou seicyo (Crystal Growth). Syokabo, Tokyo (1977).

[19] A.R. Roosen and J.E. Taylor, Modeling crystal growth in a diffusion field using fully faceted interfaces. J. Comput. Phys. 114 (1994) 113-128.

[20] P. Rybka, A quasi-steady approximation to an integro-differential model of interface motion. Appl. Anal. 56 (1995) 19-34.

[21] P. Rybka, A crystalline motion: uniqueness and geometric properties. SIAM J. Appl. Math. 57 (1997) 53-72.

[22] I. Sunagawa, K. Narita, P. Bennema and B. van der Hoek, Observation and interpretation of eccentric growth spirals. J. Crystal Growth 42 (1977) 121-126.

[23] J.E. Taylor, Crystalline variational problem. Bull. Amer. Math. Soc. 84 (1978) 568-588.

[24] J.E. Taylor, Constructions and conjectures in crystalline nondifferential geometry, in Differential Geometry (A symposium in honour of Manfredo do Carmo), B. Lawson and K. Tenenblat Eds., Longman, Essex (1991) 321-336.

[25] J.E. Taylor, Motion of curves by crystalline curvature, including triple junctions and boundary points. Proc. Sympos. Pure. Math. 54 (1993) 417-438.

[26] J.E. Taylor, Surface motion due to crystalline surface energy gradient flows, in Elliptic and Parabolic Methods in Geometry, B. Chow, R. Gulliver, S. Levy, J. Sulliva and A.K. Peters Eds., Massachusetts (1996) 145-162.

[27] J.J. Tyson and J.P. Keener, Singular perturbation theory of traveling waves in excitable media. Physica D 32 (1988) $327-361$.

[28] T. Ushijima and S. Yazaki, Convergence of a crystalline algorithm for the motion of a closed convex curve by a power of curvature $v=\kappa^{\alpha}$. Preprint (1997). 